\title{
EchoGéo
}

\section{Les dimensions géopolitiques du processus de rénovation politique en Equateur}

\section{Alexis Sierra}

\section{(2) OpenEdition}

\section{Journals}

Édition électronique

URL : https://journals.openedition.org/echogeo/2213

DOI : 10.4000/echogeo.2213

ISSN : 1963-1197

\section{Éditeur}

Pôle de recherche pour l'organisation et la diffusion de l'information géographique (CNRS UMR 8586)

Référence électronique

Alexis Sierra, "Les dimensions géopolitiques du processus de rénovation politique en Equateur», EchoGéo [En ligne], 3 | 2007, mis en ligne le 13 mars 2008, consulté le 01 août 2021. URL : http:// journals.openedition.org/echogeo/2213; DOI : https://doi.org/10.4000/echogeo.2213

Ce document a été généré automatiquement le 1 août 2021

EchoGéo est mis à disposition selon les termes de la licence Creative Commons Attribution - Pas d'Utilisation Commerciale - Pas de Modification 4.0 International (CC BY-NC-ND) 


\title{
Les dimensions géopolitiques du processus de rénovation politique en Equateur
}

\author{
Alexis Sierra
}

1 Le 26 novembre 2006, Rafael Correa a été élu président de la République au $2^{\mathrm{e}}$ tour d'une scrutin particulièrement disputé. Il est le $12^{\mathrm{e}}$ président du pays depuis la restauration de la démocratie multipartiste en 1978 et le 7e en 10 ans $^{1}$. En effet, depuis 1996, le pays a connu une instabilité politique chronique sur fond de crise économique. L'élection de Rafael Correa prolonge la série d'élections qui en Amérique latine a porté des présidents marqués à gauche depuis celle de Lagos au Chili en 2000 et de Lula au Brésil en 2003. La position de Correa dans cet échiquier politique latino-américain est intermédiaire entre la social-démocratie du président brésilien et le nationalisme de gauche $^{2}$ de Hugo Chávez, ce dernier ayant largement soutenu sa candidature. Rafael Correa, dont la popularité reste grande, est dans une situation encore fragile. Les élections législatives concomitantes à l'élection présidentielle ne lui avaient pas donné de majorité au congrès. Le mouvement qu'il a créé pour porter sa candidature, Alianza País, n'avait pas présenté de candidats aux élections législatives faute d'être un parti politique structuré et enraciné. Cependant, comme Hugo Chávez au Venezuela, il a réussi à lancer un processus de révision constitutionnelle qui lui a apporté une très forte légitimité. Le référendum organisé en avril 2007 approuvant ce processus puis le raz-de-marée des partisans de Correa à l'assemblée constituante élue en novembre de la même année se sont transformés en véritable plébiscite pour le président. C'est pourquoi Rafael Correa pourrait casser le cercle vicieux de l'instabilité politique de la dernière décennie et donner des marges de manœuvre à un pays tiraillé entre son alliance avec le Etats-Unis et son anti-américanisme populaire. Aussi, pour comprendre le mouvement qui parcourt aujourd'hui l'Equateur, ne suffit-il pas de situer l'élection du nouveau président dans le contexte latino-américain favorable à la gauche : nous devons l'analyser dans un contexte national tourmenté depuis une dizaine d'années et en tenant compte de la géopolitique intérieur et extérieure de ce pays de $283000 \mathrm{~km} 2$ et de 13,4 millions d'habitants (2005) (Figure 1). Cette courte étude ne prétend pas à 
l'exhaustivité et repose pour partie sur les résultats électoraux publiés durant l'année 2007 par le Tribunal Suprême Electoral équatorien. Elle vise avant tout à donner quelques repères et un éclairage sur la situation politique de ce pays.

Figure 1 - Les provinces équatoriennes et leurs poids démographique (en \% du total national)

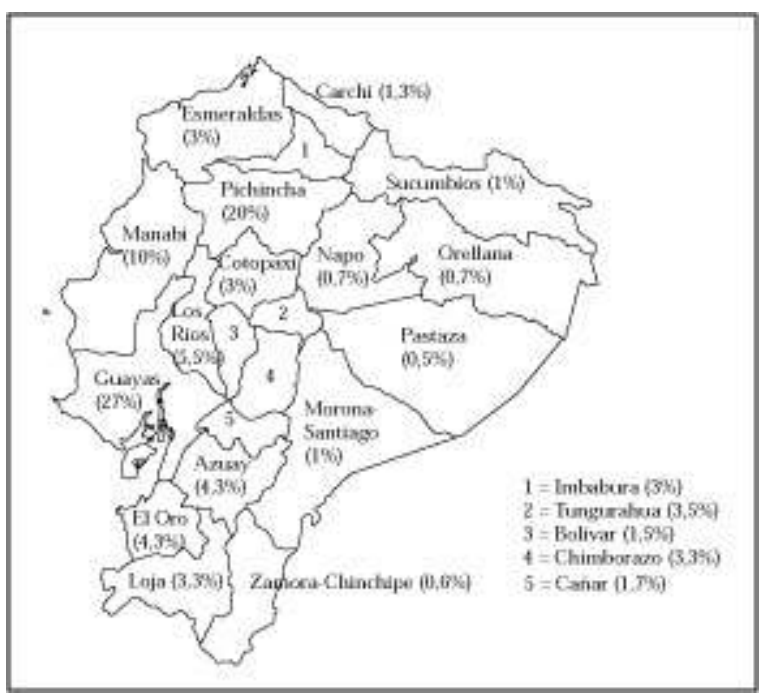

Le régionalisme et le taux d'urbanisation comme facteurs discriminants du vote au premier tour

2 Les élections présidentielles de 2006 ont vu s'affronter 13 candidats au premier tour (Figure 2). Au second tour, Rafael Correa, candidat indépendant, a dû affronter Álvaro Nobóa, candidat du parti populiste de droite Partido Renovador Institucional Acción Nacional (PRIAN). Le premier enseignement de ce scrutin tient dans le taux d'abstention, un des plus élevés jamais enregistrés. En effet, $28 \%$ des inscrits ne se sont pas déplacés alors même que le vote est obligatoire pour les personnes alphabétisées de moins de 65 ans. En l'absence d'enquête, nous ne pouvons que formuler des hypothèses à partir des commentaires locaux et évoquer d'une part, la lassitude et le scepticisme face à la classe politique, la forte croissance du nombre d'émigrés d'autre part ${ }^{3}$. 
Figure 2 - Résultats du premier tour des élections présidentielles de 2006

\begin{tabular}{|c|c|c|c|}
\hline Candidat & Pard & $\begin{array}{l}\text { Résultats au } \mathrm{I}^{*} \\
\text { tour }\end{array}$ & $\begin{array}{l}\text { Nombre de provinces où le candidat arrive en tẻte } \\
\text { (total 21) }\end{array}$ \\
\hline Alvaro Nobos & PRIAN & $26.8 \%$ & 4 ( 3 sur la Costa. I sur la Sierra) représentant $42 \%$ \\
\hline Rathel Correa & Alianza PAIS & $228 \%$ & $\begin{array}{l}5 \text { ( } 4 \text { sur la Slerra, I sur la Costa) représentant } 35 \% \text { de } \\
\text { la population nationaic }\end{array}$ \\
\hline Gilmar Gutierrez & PSP & $17,4 \%$ & $\begin{array}{l}12 \text { (toutes les provinces amazoniennes, } 6 \text { provinces de } \\
\text { la Siera et I de la Costa) représentam } 23 \% \text { de la } \\
\text { population mationale }\end{array}$ \\
\hline Leon Roldos & ID & $14.8 \%$ & \\
\hline Cynchia Viteri & PSC & $9,6 \%$ & \\
\hline Luis Macas & MUPP-NP & $2.2 \%$ & \\
\hline Fernando Posero & PRE & $2,1 \%$ & \\
\hline Marco Prosño & MRD & $1,4 \%$ & \\
\hline Luis Villads & MPD & $1.3 \%$ & \\
\hline Jaime Damerval & CFP & $0.5 \%$ & \\
\hline Marcelo Larrea. & Ind. & $0.4 \%$ & \\
\hline Lenin Torres: & Ind. & $0.3 \%$ & \\
\hline Carlos Sanay & Ind. & 0,25 & \\
\hline
\end{tabular}

Source : Tribunal Supremo Electoral, Equateur

Les résultats par province montrent la persistance du régionalisme dans la géographie électorale (figure 3). La géographie équatorienne est en effet porteuse d'un dualisme entre les provinces côtières, la Costa (50\% de la population totale aujourd'hui), et les provinces andines de la Sierra (45\% de la population) (Deler, 1981). Ce dualisme est à nuancer par la croissance des provinces amazoniennes de l'Oriente $(5 \%$ de la population) et par les différences internes à la Sierra et à la Costa. Les résultats par province masquent notamment de forts contrastes intraprovinciaux en particulier entre les métropoles régionales et le reste de la province. Nous n'avons pas pu avoir accès pour l'instant aux résultats par canton ou parroquia ${ }^{4}$ qui permettraient d'affiner la géographie électorale. Cependant, les résultats des élections présidentielles montrent la vivacité de la représentation duale voire tripartite du pays : la Costa pour Nobóa, la Sierra pour Correa, l'Oriente pour Gutiérrez. En effet, le premier tour des élections montre que Álvaro Nobóa l'emporte dans toutes les provinces côtières à l'exception de El Oro. Rafael Correa n'arrive en tête que dans la Sierra notamment dans ses deux provinces les plus peuplées, le Pichincha et l'Azuay. En dehors de cette région andine, il ne l'emporte que dans El Oro. Enfin, Gilmar Gutiérrez a créé la surprise par sa troisième position. Il devance ses concurrents dans toutes les provinces amazoniennes. Cependant, il trouble un schéma qui serait simplement tripartite : en effet, il l'emporte également dans les 5 provinces centrales de la Sierra et dans la province côtière de Los Ríos qui jouxte cet ensemble andin. 
Figure 3 - Candidats arrivés en tête au premier tour des élections présidentielles

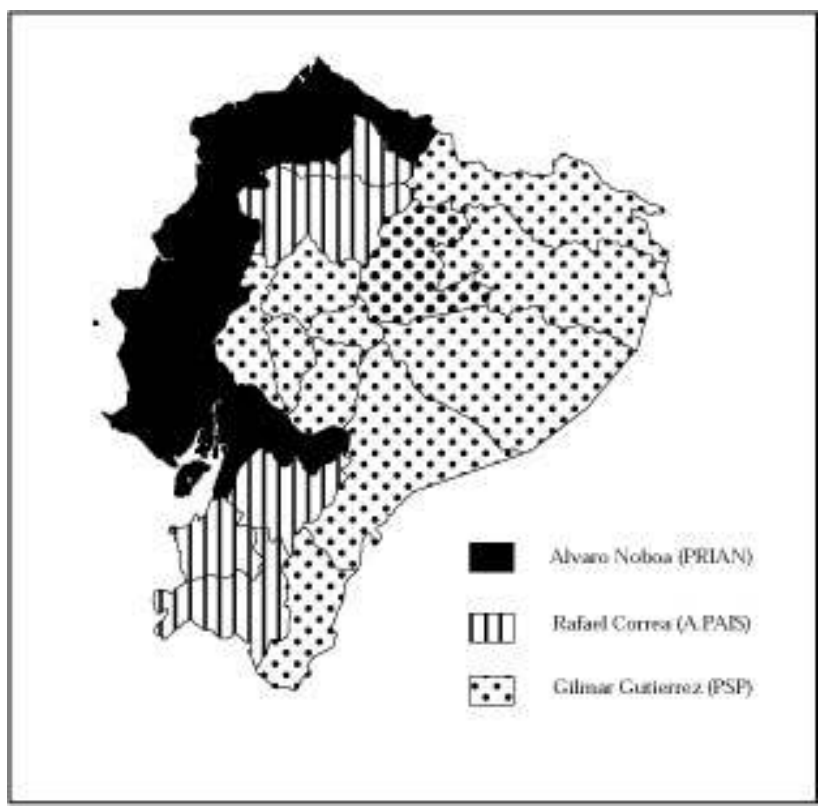

Figure 4 - Taux d'urbanisation des provinces de la Sierra dans lesquelles Correa et Gutierrez arrivent en tête et de El Oro (classé en fonction du taux d'urbanisation

\begin{tabular}{|l|c|c|}
\hline Province & Taux d'urbanisation & Candidat arrive en téte \\
\hline E Oro & $76 \%$ & Correa \\
Pichincha & $72 \%$ & Correa \\
Aziay & $52 \%$ & Correa \\
Imbabura & $50 \%$ & Correa \\
Loja & $45 \%$ & Correa \\
Tungurahta & $43 \%$ & Gutierrez \\
Chimborazo & $3 \% \%$ & Gutierrez \\
Cotopaxi & $27 \%$ & Gutierrez \\
Bolivar & $26 \%$ & Gutierrez \\
\hline Somurze
\end{tabular}

4 Aussi, la géographie électorale de la Sierra est-elle intéressante à regarder de près pour saisir la faiblesse relative de Correa. Le taux d'urbanisation semble en effet fortement discriminant: il y a un partage parfait des choix effectués au premier tour entre provinces urbaines et provinces rurales (figure 4). Ainsi, les provinces de la Sierra où Rafael Correa l'emporte sont celles qui ont le taux d'urbanisation le plus élevé, compris entre $45 \%$ à $72 \%$; inversement, les provinces les plus rurales ont préféré Gilmar Gutiérrez (taux d'urbanisation compris entre $26 \%$ et $43 \%$ ). Le cas de la province de El Oro semble confirmer que l'électorat du président élu semble dès le départ plus urbain. Cette province côtière est celle qui a le taux d'urbanisation le plus élevé du pays (76\%) après le Guayas. Or, il s'agit de la seule province non andine qui ait placé R. Correa en tête au premier tour. L'électorat de Gilmar Gutiérrez semble en revanche plutôt rural : outre dans les provinces les plus rurales de la Sierra, ce candidat est victorieux dans les provinces amazoniennes où les taux d'urbanisation sont compris entre $30 \%$ et $44 \%$. Cependant, le taux d'urbanisation permet surtout d'analyser les différences au sein d'une même région. Il ne peut en effet expliquer l'ensemble de la géographie électorale équatorienne puisque Álvaro Nobóa l'emporte dans les provinces côtières qui sont majoritairement urbaines. 
Le rôle du régionalisme et de l'incarnation de la diversité nationale au second tour des élections

5 Au second, tour, c'est davantage le dualisme Sierra/Costa qui est réaffirmé (figure 5 et 6). Les provinces côtières, à l'exception de El Oro, ont donné leurs suffrages à Álvaro Nobóa. Celui-ci maintient ainsi ses positions initiales. Il perd cependant le Carchi, province de la Sierra, où il arrivait pourtant en tête au premier tour. Cet exemple montre que, dans la Sierra, Rafael Correa a su attirer à lui l'électorat des différentes forces politiques en s'appuyant notamment sur le rejet de Álvaro Nobóa. Le président élu conserve non seulement son avantage dans les provinces où il arrivait en tête au premier tour mais il l'emporte aussi dans toutes les provinces où Gilmar Gutiérrez arrivait en tête, à l'exception du Napo. Le vote de rejet à l'encontre du candidat du PRIAN semble être ainsi confirmé.

Figures 5 et 6 - A gauche : Provinces dans lesquelles Alvaro Noboa (PRIAN) est arrivé en tête du second tour. A droite: Provinces dans lesquelles Rafael Correa est arrivé en tête au second tour

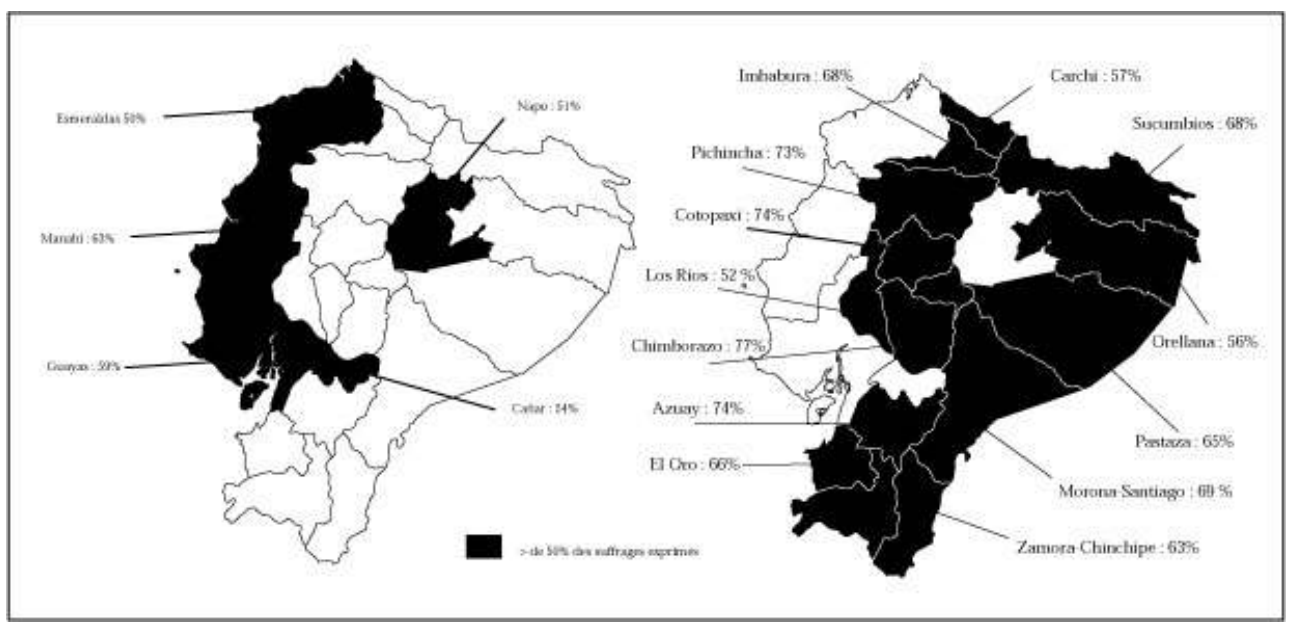

6 Représentant clairement les intérêts de l'oligarchie guayaquilègne, Álvaro Nobóa est anciennement implanté dans le Guayas et le Manabí par ses activités, par les réseaux qu'il y a tissé et les clientèles qu'il y a créées. Riche entrepreneur surnommé le « tzar de la banane ", ce milliardaire en dollars possède une dizaine de plantations géantes et une centaine d'entreprises dont une banque, deux compagnies d'assurances, quatre entreprises de navigation maritime et plusieurs entreprises agroalimentaires. Son empire, le Grupo Nobóa, est une firme multinationale qui contrôle $9 \%$ du marché mondial de la banane. Déjà candidat en 1998, il représentait alors le Partido Roldosista Ecuatoriano (PRE), le parti populiste du président corrompu et destitué Abdalá Bucaram. Son score, de $49 \%$ face au démocrate-chrétien Jamil Mahuad, a montré combien le discours populiste a eu un écho dans un pays en crise. Candidat malheureux face à Lucio Gutiérrez en 2002, il a de nouveau surpris en 2006 en arrivant en tête du premier tour des élections présidentielles avec près de $27 \%$ des suffrages.

7 Cependant, avec $43 \%$ des suffrages au second tour, il réalise là son plus faible score malgré la débauche de moyens utilisés. Ses campagnes sont en effet exemplaires du clientélisme et du populisme latino-américain. Grâce à sa fortune personnelle, il a selon la presse nationale dépensé en moyenne 9,5 dollars par vote contre 1,65 dollars pour Rafael Correa. Il a ainsi distribué des chaises roulantes pour les handicapés et des milliers de T-shirts, facilité des prêts, distribué de l'argent liquide ${ }^{5} .$. Son style fut 
d'emblée emphatique, se mettant en scène, allant jusqu'à s'agenouiller pour implorer le seigneur tout en développement un credo ultra-libéral. Le fait qu'il ait développé son activité politique dans la partie la plus peuplée du pays (la Costa représente $50 \%$ de la population) lui donnait un avantage qui s'est traduit par sa position au premier tour. La plupart des présidents élus étaient soit représentants élus, soit originaires à un titre ou un autre de la Costa. L'unique président élu dont les attaches étaient exclusivement de la Sierra, Rodrigo Borja, a dû remporter 20 provinces sur 21 en 1988 pour pouvoir devenir président face à un candidat de Guayaquil. Álvaro Nobóa avait donc une assise dans la région majoritaire et des réseaux dans le reste du pays. En revanche, il n'est pas parvenu à incarner la diversité de la nation comme a pu le faire son compétiteur. Il a globalement eu du mal à rassembler au-delà de son camps comme le montrent les écarts de voix par provinces entre les deux finalistes : là où R. Correa l'emporte, c'est avec un écart de voix très nettement supérieur à l'écart constaté dans les provinces où A. Nobóa l'emporte. Prenons le cas des deux provinces les plus peuplées: si le Guayaquilénien réalise $59 \%$ dans le Guayas, R. Correa atteint 73 \% dans le Pichincha. Álvaro Nobóa opère son record dans le Manabí avec $63 \%$ des suffrages, mais son compétiteur dépasse ce score dans 13 provinces pour atteindre $77 \%$ dans le Chimborazo. Si l'écart national (46\%-56\%) n'est pas aussi important entre les deux candidats, c'est en raison du poids démographique respectif des différentes provinces, le Guayas et le Manabí par exemple représentent à eux seuls $37 \%$ de la population nationale.

Rafael Correa a eu le double avantage d'être guayaquilègne par sa naissance, son enfance et ses études et d'être quiténien par sa résidence et son lieu d'exercice professionnel. Au-delà de son positionnement idéologique, il pouvait donc davantage apparaître comme pleinement équatorien. Il a également su attirer les électorats de Gilmar Gutiérrez (arrivé $3^{e}$ au premier tour) et du MUPP-NP hostiles à l'oligarchie de Guayaquil et bien implantés dans les provinces amazoniennes. Il est également apparu comme un homme neuf face à Álvaro Nobóa qui en était à sa troisième élection. Candidat pour la première fois, Rafael Correa n'a été ministre de l'économie que quatre mois en 2005. Il incarnait enfin une classe moyenne certes urbaine et fortement diplômée mais sans luxe ostentatoire. Il se distinguait ainsi du milliardaire représentant une forme d'élite contrôlant le pouvoir économique et étroitement liée aux acteurs étrangers. Idéologiquement, le président élu s'est fait connaître pour ses positions antiaméricaines, radicalement à gauche parce qu'interventionniste, mâtinées de catholicisme social ${ }^{6}$, autant de caractéristiques qui pouvaient rassurer une nation qui se méfie des Etats-Unis et considère le christianisme comme source de morale et d'identité. Ainsi, si son discours radical a de quoi effrayer les soutiens du Partido Social Cristiano (PSC), la carte électorale semble montrer qu'une partie de l'électorat de ce parti s'est reporté sur son nom: c'est particulièrement net dans l'Azuay, province réputée conservatrice dans le domaine social et dans laquelle le PSC reste encore assez bien implanté (figure 7). 
Figure 7 - Vote en faveur du Partido Social Cristiano

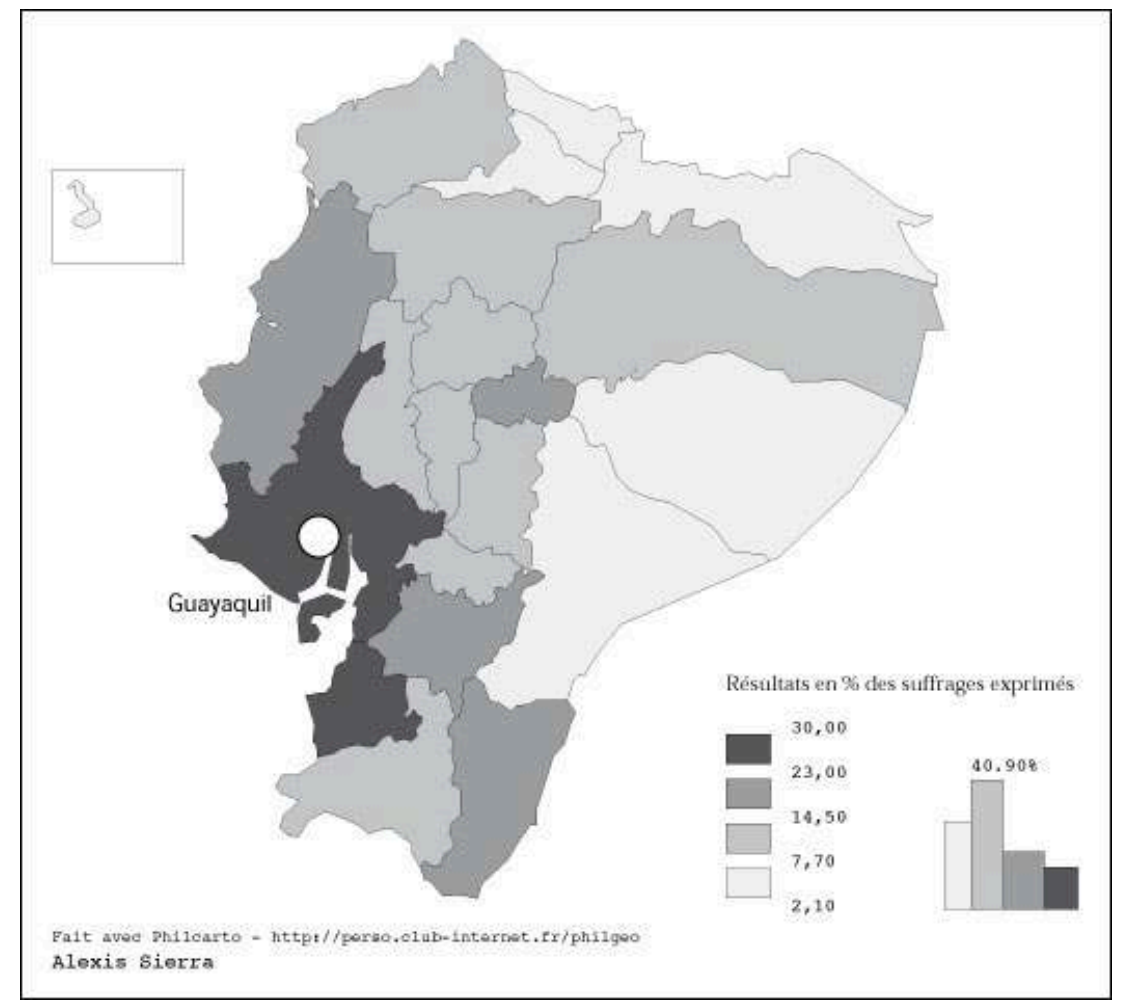

Un monde politique équatorien en mouvement

L'évolution du poids des partis traditionnels est en effet un autre des enseignements de ces élections. Depuis le retour de la démocratie, 4 ou 5 partis ont dominé le pays : la Izquierda Democrática (ID, social-démocrate), la Démocracia Popular (DP, Démocratechrétien), le Partido Social-Cristiano (PSC, droite) et le Partido Roldosista Ecuatoriano (PRE, droite populiste $)^{7}$. La frise montre que ces partis ont alternativement gagné la présidence de la République jusqu'en 1998. En revanche, les deux derniers présidents élus ainsi que leur vice-président sont des candidats indépendants : voir la figure 8 cidessous.

Successions presidentielles depuis la crise de 1996

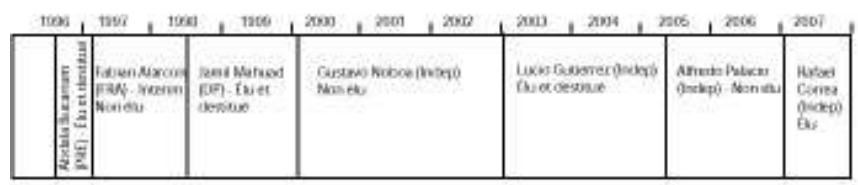

Successions présidentielles depuis la fin de la dictature militaire

\begin{tabular}{|c|c|c|c|c|c|}
\hline $\begin{array}{l}\text { Fadeb } \\
\text { jersuser }\end{array}$ & 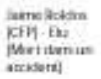 & 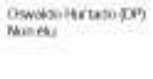 & 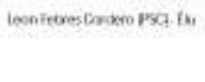 & 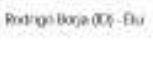 & 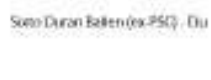 \\
\hline
\end{tabular}

En 2006, nous retrouvons ainsi la configuration vénézuelienne dans laquelle Hugo Chávez a été élu, à savoir une victoire par opposition aux partis qui s'étaient jusque-là partagés le pouvoir. Les trois candidats ayant reçu le plus de suffrages au premier tour des élections présidentielles ont créé leur propre mouvement politique, rompant avec les partis traditionnels. Ainsi, Álvaro Nobóa, Rafael Correa et Gilmar Gutiérrez totalisent les $2 / 3$ des votes alors que Leon Roldos soutenu par la ID et Cynthia Viteri 
candidate du PSC ne recueillaient à eux deux qu'un quart des votes. Le reste des suffrages est éclaté entre 8 autres candidats qui ont recueilli moins de $3 \%$ chacun (figure 1). Cette évolution semble confirmer une forme de rejet du système politique traditionnel.

Figure 9 - Vote en faveur de la Izqueida

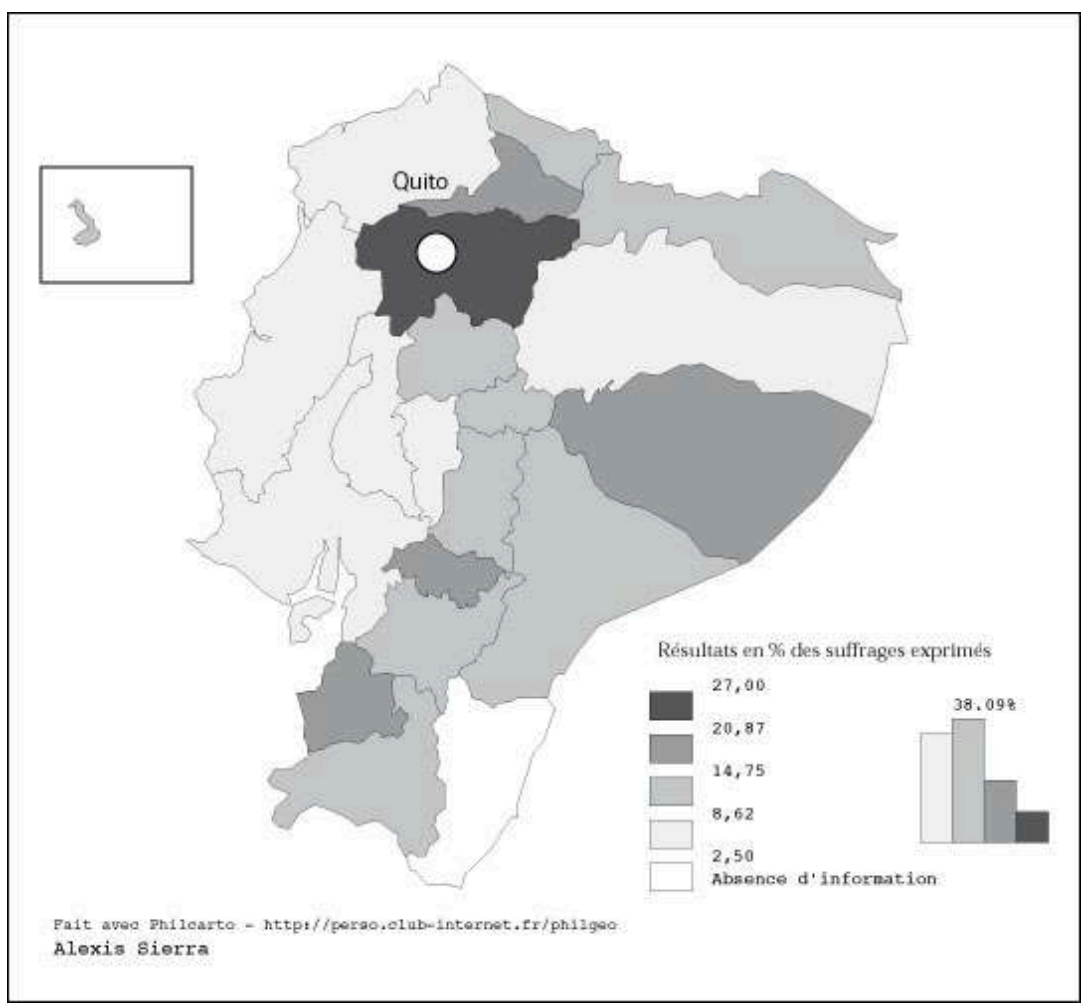


Figure 10 - Vote en faveur du Partido Sociedad Patriotica

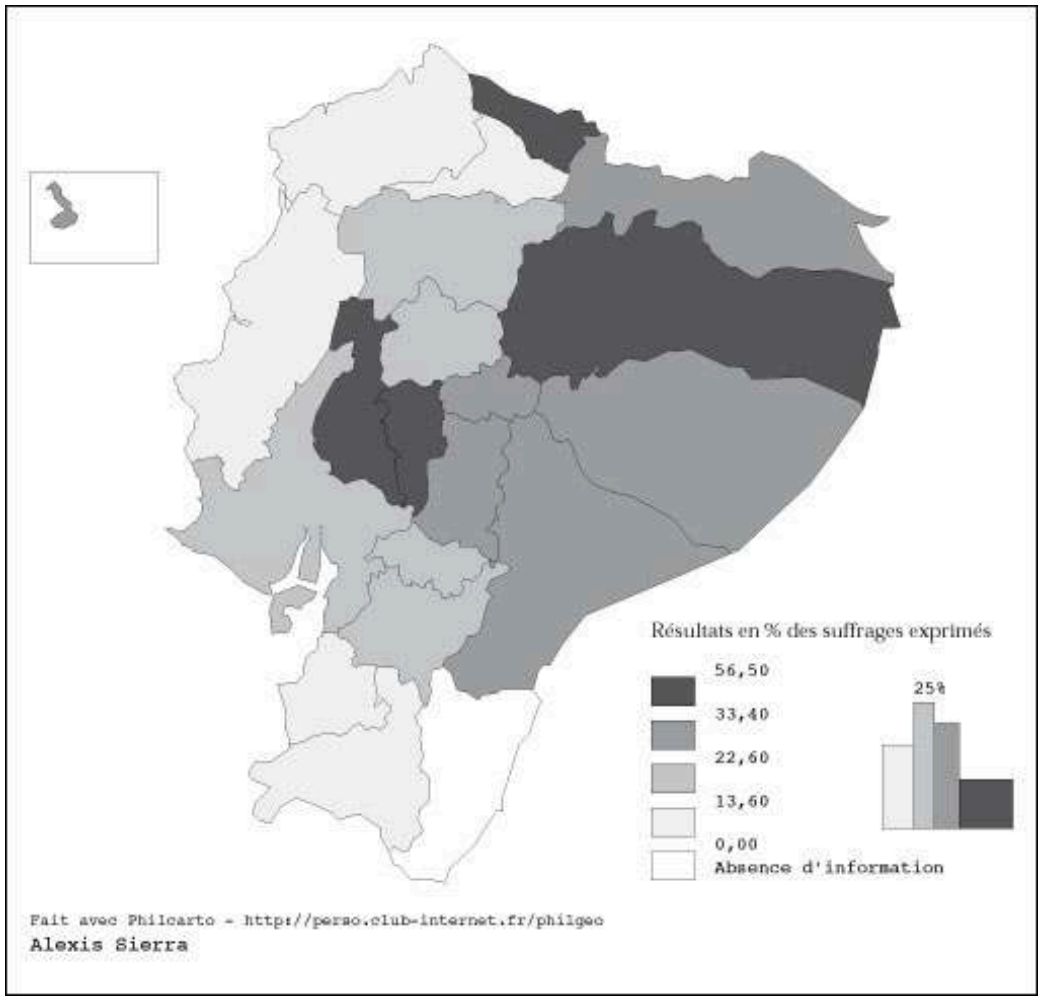

Figure 11 - Vote en faveur du Mouvement patchakutik Nuevo Pais

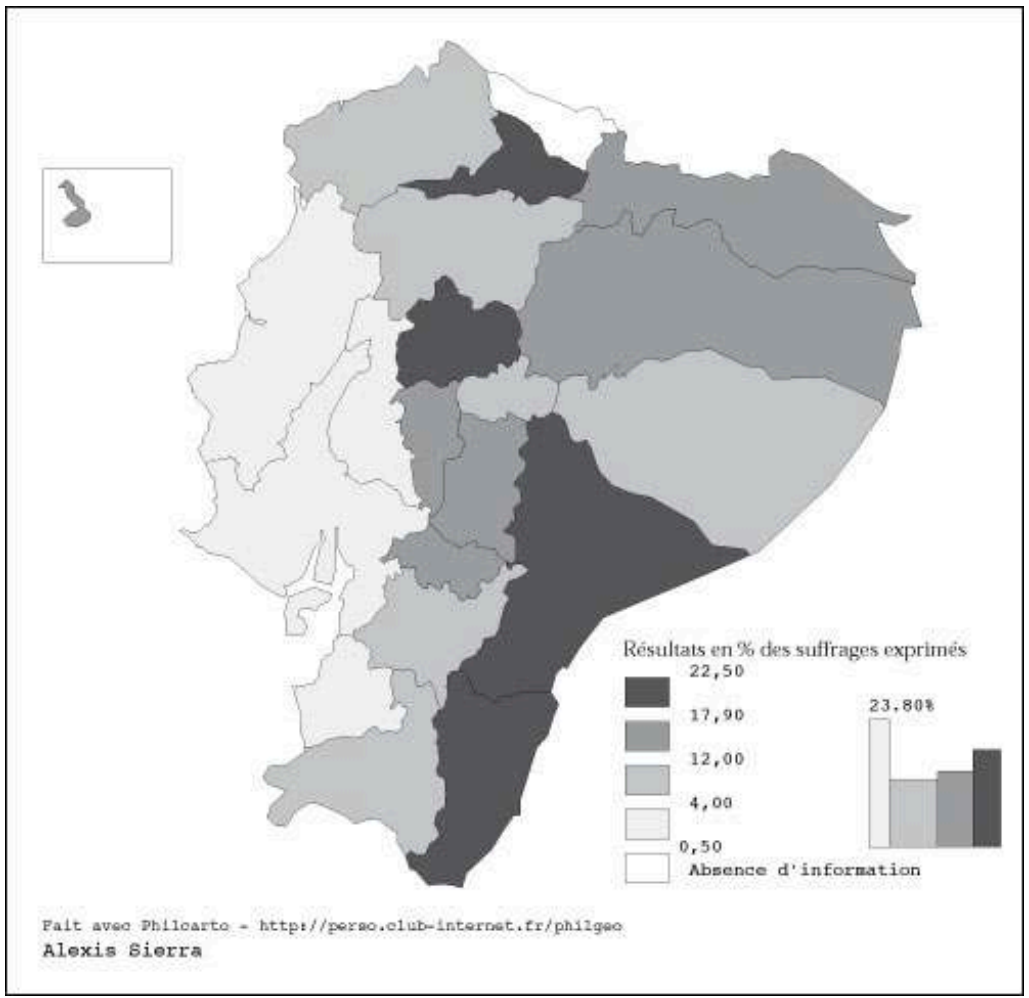

11 Les élections législatives aboutissent à des évolutions similaires. Jusqu'en 2002, les 4 partis mentionnés, ID, DP, PDC, PRE contrôlaient l'assemblée. A la suite des dernières 
élections, il est contrôlé par deux partis, le Partido Renovador Institucional Acción Nacional (PRIAN) et le Partido Sociedad Patriotica del 21 de enero (PSP), tous deux de création récente. Le premier a été créé en 2000 par Álvaro Nobóa en faisant scission avec le PRE dont il capte aujourd'hui l'essentiel de l'électorat. Le second a été créé par Lucio Gutiérrez à la suite du soulèvement de janvier 2000 pour accéder à la présidence en 2003. L'existence de ces deux partis dominants montre la complexité de la situation électorale et législative que doit gérer Rafael Correa. Du fait de sa forte implantation dans la Sierra, le PSP a la même base électorale que Rafael Correa (figure 10). Quant aux autres partis, ils résistent essentiellement dans leurs fiefs d'origine, à savoir la région de Quito pour la Izquierda Democrática (figure 9) et celle de Guayaquil pour le Partido Social Cristiano (figure 7). Les résultats de ces deux partis sont très éclairants du régionalisme passé et de son éventuelle évolution. Le PSC domine dans le Guayas, la province la plus peuplée du pays (27\% de la population nationale). Il dirige la municipalité de Guayaquil, la métropole économique. Léon Febres Cordero, l'homme fort du parti durant les 25 dernières années fut président de la République (1984-1988) puis maire de Guayaquil (1992-2000). Son assise locale et régionale permet au PSC de conserver 5 sièges sur 18 pour le Guayas. À cela s'ajoute sa position majoritaire dans la province bananière de El Oro. Il faut également souligner la forte influence du PSC dans la province côtière du Manabí, la $3^{\mathrm{e}}$ la plus peuplée avec 1,2 millions d'habitants. Dans les provinces côtières, il a été fortement concurrencé par le PRE puis aujourd'hui par le PRIAN de Álvaro Nobóa. Ainsi, aux législatives le PRIAN domine la province du Manabí, l'une des plus peuplées mais également l'une des plus pauvres du pays. Enfin, le PSC garde une forte influence dans des provinces de la Sierra: le Tungurahua (17,5\%), l'Azuay (15\%), la $5^{\mathrm{e}}$ la plus peuplée, Loja (13\%), et le Cotopaxi (11\%). Dans chacune d'elles, le PSC sauve un siège de député. Cette implantation électorale explique que la diminution du nombre d'élus du PSC soit moindre que celle de son équivalent à gauche, la ID. Ce dernier avait une assise régionale sur l'ensemble de la Sierra avec des développements marginaux sur la Costa et l'Oriente. Sa géographie électorale est beaucoup plus polarisée sur une seule province, celle du Pichincha. Certes, cette dernière avec 2,4 millions d'habitants, est la $2^{e}$ du pays. Elle est la région-capitale avec tout ce que cela implique d'acteurs et de réseaux influents. Cependant, dans les autres provinces de la Sierra, la ID a progressivement vu son influence s'éroder au profit du MUPP-NP (Imbabura, Chimborazo, Cotopaxi) et du PSP (Chimborazo, Bolívar, Tungurahua, Cañar). Au total, l'électorat de la Sierra est davantage divisé en plusieurs partis de poids équivalents alors que celui de la Costa se divise essentiellement entre le PRIAN et le PSC. Cet éclatement aboutissait depuis des années à une absence de majorité au Congrès. À cet éclatement, s'est ajoutée en 2006 l'absence d'élus représentant le nouveau président de la république.

12 Les résultats aux élections législatives de 2006 montraient donc l'existence d'un obstacle parlementaire pour le nouveau président. C'est pourquoi, le processus de réforme constitutionnelle engagé vise à contourner ce qui serait un frein à sa politique. Rafael Correa a ainsi organisé le 15 avril 2007 un référendum sur la tenue d'une assemblée constituante, référendum qui, avec plus de $78 \%$ de oui, s'est transformé en plébiscite. Fort de ce score, il a engagé le processus: de nouvelles élections ont été organisées pour mettre en place cette assemblée qui a les pleins pouvoirs pour modifier la constitution et l'organisation politique du pays. Les élections du 30 septembre 2006 lui ont donné une victoire écrasante : avec $69 \%$ des suffrages, son mouvement, Alianza País remporte 73 sièges sur 130 ! Le PSP et le PRIAN n'atteignent pas $14 \%$ à eux deux et 
sauvent 26 sièges alors qu'aux élections législatives de 2006 ils totalisaient un tiers des suffrages et obtenaient 52 députés sur 100. Les deux anciens partis, le PSC et plus encore la ID, déjà très affaiblis aux élections de 2006, sont laminés. Ils n'arrivent à sauver que huit sièges au total, dans leurs bastions traditionnels, le Guayas pour le premier et le Pichincha pour le second. Il semble d'ailleurs que l'électorat de la ID comme celui du MUPP-NP se soit porté facilement sur les candidats de Alianza País confirmant l'ancrage de Rafael Correa sur la Sierra. Cette victoire électorale permet à Rafael Correa de dissoudre le Congrès, l'assemblée constituante la remplaçant. Celle-ci doit rédiger la nouvelle constitution qui sera soumise à référendum en 2008. De nouvelles élections générales doivent avoir lieu avec son adoption. Ici encore on retrouve le processus politique mis en place au Venezuela sans qu'il faille faire de ce dernier pays un modèle importé, Rafael Correa affirmant lui-même que le «socialisme du $\mathrm{XXI}^{\mathrm{e}}$ siècle en Equateur ne sera pas celui de Chávez ${ }^{8}$. Cette réforme constitutionnelle montre cependant que trouver un remède à l'instabilité politique et restaurer l'autorité publique nationale peuvent apparaître comme prioritaires à? Une majorité des citoyens.

La stabilisation politique, un défi à relever

13 L'histoire des dix dernières années montre en effet le défi que doit relever Rafael Correa, non seulement pour trouver des relais législatifs dans les différentes provinces mais aussi pour redonner au pays une stabilité politique perdue depuis 1996.

Comme indiqué en introduction, à partir de 1996, le pays entre dans une période de turbulence qu'éclaire la chronologie des présidences successives (figure 7). Mise ainsi en perspective, la présidence de Rafael Correa apparaît d'autant plus comme un défi qu'aucun président élu depuis 1996 n'a pu finir son mandat. Trois présidents élus (A. Bucarram, J. Mahuad, L. Gutiérrez) ont été destitués sur fond de crise économique, financière, sociale et morale. Trois présidents (F.Alarcón, G. Nobóa, L.A. Palacio) n'ont pas été élus et ont succédé à ces présidents destitués. Faiblement légitimes et dépendants du jeu des partis au Congrès, ils n'ont pas eu l'assise politique que confère une élection directe pour mener des réformes profondes. Ce furent des présidences de gestion qui n'ont fait que retarder la rénovation du système politique et économique. Leur mandat fut cependant aussi long que celui des présidents élus montrant que la légitimité issue du Congrès dont ils dépendaient pouvait être équivalente à celle du suffrage universel direct. Cette période est d'autant plus surprenante que du retour à la démocratie en 1978 jusqu'à 1996, l'alternance politique a fonctionné normalement avec 5 présidents dont 4 élus 9 . La situation se renverse en 1996 et la présidence de Abdalá Bucarram, la plus courte depuis le retour de la démocratie. Très largement contesté, Abdalá Bucarram est destitué par le Congrès qui, anticonstitutionnellement, élit Fabian Alarcón ${ }^{10}$ pour lui succéder. Cette manière de faire face à la crise allait créer un précédent : en 2000 et en 2005, le Congrès destitue le président ou le contraint à démissionner sous la pression populaire. En 1997, Fabian Alarcón chercha cependant à se légitimer en organisant un plébiscite qu'il remporte. Il organisa alors des élections pour mettre en place une assemblée constituante. La comparaison avec la situation actuelle s'arrête-là. En effet, à l'époque, le Congrès, dominé par les partis traditionnels, parvint à limiter le pouvoir de cette constituante. La réforme constitutionnelle ne devait pas changer l'équilibre des pouvoirs : elle se borna essentiellement à régler la question de la succession présidentielle et à établir de nouveaux droits et devoirs. Quand Jamil Mahuad est élu en 1998, aucune réforme de fond n'a été engagée pour faire face à la crise financière dont la violence prend les autorités de court. Quand il décide 
de dollariser le pays à marche forcée, le train de mesures est si impopulaire que les manifestations parviennent à bloquer le pays et à provoquer le rapprochement entre une partie de l'armée et les communautés indiennes. La marche des mouvements indigènes aboutit ainsi à assaillir le Congrès et à la mise en place d'un comité de salut public dirigé par le président de la confédération des nationalités indigènes d'Equateur (CONAIE) et par le colonel Lucio Gutiérrez (Gallegos, 2000). Ce dernier crée le PSP et remporte les élections de 2002 en prônant la rupture. Cependant, il semble tourner le dos à son programme une fois élu en acceptant la dollarisation effectuée et en suivant les préceptes des institutions internationales. Il engage une privatisation du secteur pétrolier. Cette décision mêlée à des accusations de népotisme déclanche un mouvement similaire à celui qui l'avait fait connaître mais qui cette fois-ci provoque sa destitution. Rafael Correa devient ainsi le troisième président élu dans un contexte de crise, de grande défiance des pouvoirs publics et d'affaiblissement de la légitimité présidentielle du fait de ces destitutions à répétition. Dans ce bref historique, Quito, comme capitale est le décor de ces convulsions. Si les mouvements de contestation convergent vers Quito, c'est plus généralement l'ensemble de la Sierra et des provinces amazoniennes qui sont traversés par l'expression de la crise sociale. La création récente de partis politiques sur la Sierra (MUPP-NP, PSP, Alianza PAÍs) trouve là son origine. Cette situation géopolitique traduit également le rôle de deux protagonistes : l'armée et la communauté indienne.

Le rôle de l'armée

15 L'armée, comme dans beaucoup d'Etats latino-américain, a été un protagoniste dominant de la vie politique. L'Equateur a connu une dictature militaire de 1973 à 1978. Avec la démocratie, l'armée a conservé une très grandes influence politique et économique (Moncayo, 1995 ; collectif, 1996). Elle contrôle aujourd'hui de nombreuses entreprises de la finance (banco Rumiñahui) à l'aviation civile (TAME) en passant par le secteur touristique (Amazónica sa). Elle dirige en particulier la HoldingDine qui détient des actions dans l'industrie sidérurgique, d'extraction, du bâtiment, de l'armement, du textile, de l'agroalimentaire ainsi que des plantations de banane et de fleurs et des compagnies de navigation maritime. En 1997, lors de la destitution de Abdalá Bucararm, c'est l'armée qui a assuré la médiation entre les différents protagonistes, couvert le départ du président déchu et assuré la transition. Le chef d'Etat major de l'époque, Paco Moncayo, est depuis devenu maire de Quito. L'armée bénéficie également d'une certaine confiance auprès de la population car elle est l'une des rares institutions ayant assuré la continuité de l'Etat. L'armée a également un rôle d'intégration nationale grâce au service militaire. C'est ce qui explique sa capacité de médiation en cas de crise ou comme en 2000 le fait qu'elle puisse basculer du côté du soulèvement populaire. Dans ce dernier cas, la détérioration des conditions de vie des cadres intermédiaires de l'armée et des soldats provoque le glissement d'une partie de l'armée du côté de la contestation et de son rapprochement avec le mouvement indien dans une même critique de la corruption, des partis traditionnels et de l'oligarchie (Gallegos, op. cit.). Quand le colonel Lucio Gutiérrez crée le PSP, il a des relais dans l'armée notamment chez les soldats. La forte présence de l'armée dans les provinces amazoniennes, peu denses et frontalières, peut expliquer la force du PSP dans ces régions.

Les organisations amérindiennes comme nouvel acteur politique

16 Selon le recensement de 2005, les Amérindiens représentent $25 \%$ de la population. Officiellement, ils se distribuent entre peuples et nationalités. Les plus nombreuses sont 
dans la Sierra où la nationalité Quichua se compose de 13 peuples. Une dizaine de peuples amérindiens vivent en Amazonie. Dans les années quatre-vingt et quatre-vingtdix, les communautés amérindiennes cherchent à faire reconnaître leur identité et l'état d'infériorité dans lequel la colonisation puis la république les ont laissées. À cet effet, plusieurs associations indiennes créent la CONAIE en 1986. La pression qu'elles exercent aboutit à intégrer à la réforme constitutionnelle de 1998 leurs droits collectifs. Une section de la constitution est ainsi consacrée à la promotion de leur culture, de leur langue et de leurs savoirs ainsi qu'à la reconnaissance de leur organisation collective comme interlocuteur privilégié du pouvoir politique (cf. articles 83, 84, 85). En 1996, une partie des organisations indiennes crée le MUPP-NP et présente des candidats. Ici, également l'année 1996 est décisive. D'une part, pour la première fois, le MUPP-NP intervient directement dans la campagne en soutenant un candidat indépendant, le journaliste Freddy Ehlers. Celui-ci troubla fortement le jeu électoral en arrivant en $3^{\mathrm{e}}$ position. D'autre part, lors des élections législatives concomitantes, Luis Macas, président de la CONAIE, devient le premier indien élu député. Dès lors, le MUPP-NP est représenté au Congrès même s'il y reste très minoritaire. Il est essentiellement implanté dans les provinces du centre de la Sierra (figure 11) et dans une moindre mesure en Amazonie. La géographie électorale de ce parti explique pourquoi il ne peut être majoritaire tout en étant un acteur-clé dans la constitution d'alliances électorales : ses positions électorales sont en effet géographiquement très concentrées sur quelques provinces. Majoritaire, le MUPP-NP y fait toujours élire des représentants. Mais ces provinces rurales sont aussi peu peuplées. Elles envoient donc peu d'élus au parlement. Ce parti ne peut donc dépasser un rôle de parti charnière ou d'appoint. En revanche, les communautés indiennes sont une des forces majeures des révoltes politiques et sociales des dernières années. Comme mentionné plus haut, leur mobilisation fut ainsi à l'origine de la destitution de Jamil Mahuad en 2000. Si Luis Macas, le candidat de la CONAIE et du MUPP-NP n'a obtenu que 2,2 \% aux élections présidentielles de 2006, le MUPP-NP obtient en moyenne $12 \%$ aux élections législatives et son électorat s'est massivement reporté sur Rafael Correa, contribuant à sa victoire finale. Pour l'heure, cette frange de la population ne semble pas lui faire défaut. Le résultat désastreux du MUPP-NP à l'élection constituante (moins de $1 \%$ ) semble montrer que le mouvement du président parvient à canaliser à son profit les revendications mêlées des communautés indiennes et paysannes de la Sierra et de l'Amazonie.

Un enjeu géopolitique régional

17 Aujourd'hui, le nouveau président semble surmonter les tensions entre ces différentes forces internes au pays. Il doit également composer avec les influences réciproques des Etats-Unis et du Venezuela. La première est devenue traditionnelle. Il faut en effet rappeler que la politique monétaire de l'Equateur est menée par la Banque fédérale des EU depuis la dollarisation du pays en 2000 (Gastambide, Sierra, 2002). Bien qu'hostile à cette dollarisation pour des raisons de souveraineté nationale, Rafael Correa ne la remet pas aujourd'hui en cause: non seulement les acteurs économiques ne la souhaitent pas mais un consensus s'est finalement dégagé autour de ce remède à l'inflation. L'Equateur représente également un intérêt stratégique pour sa lutte contre le trafic de drogue : en effet, le pays est devenu ses dernières années un territoire de repli des narcotrafiquants et des guérilleros colombiens. L'Etat a concédé à la puissance nord-américaine une base militaire dans la ville côtière de Manta. Enfin, le pétrole des provinces amazoniennes intéresse les compagnies étasuniennes ${ }^{11}$. La peur d'une perte de souveraineté et la proximité de l'oligarchie guayaquilègne avec les intérêts nord- 
américains ont joué dans l'élection de Rafael Correa qui a largement développé un discours nationaliste du type de celui de Hugo Chávez. Le nom du mouvement politique de Rafael Correa est d'ailleurs significatif du désir de restaurer un certain nationalisme équatorien : Alianza PAÍS est en effet l'acronyme de « Mouvement pour une Patrie Fière Et Souveraine $»^{12}$. Les premiers mois de la présidence de Correa ont d'ailleurs montré que les sujets de tensions ne manquaient pas avec les Etats-Unis et son allié colombien : de la maîtrise nationale des ressources pétrolières à la mise en place d'un plan «Ecuador " pour contrebalancer le Plan «Colombia » de lutte contre la drogue, le nouveau président prend des mesures visant à restaurer la souveraineté de l'Equateur. Hugo Chávez quant à lui, voudrait faire de l'Equateur un allié dans sa politique régionale. L'Equateur a ainsi participé à la création de la Banque du Sud qui doit devenir une alternative à la Banque mondiale et à la Banque Interaméricaine de Développement. La situation politique de l'Equateur entre ainsi dans la bataille géopolitique que se livrent les administrations nord-américaines et vénézuéliennes. Toute la question est de savoir comment Rafael Correa parviendra à gouverner face à cette situation politique interne et internationale et s'il arrivera autant à mettre fin à l'instabilité politique qu'a connu le pays ses dix dernières années qu'à améliorer la vie des 14 millions d'Equatoriens.

\section{BIBLIOGRAPHIE}

Collectif, 1996, Fuerzas Armadas, desarrollo y democracia, Instituto latino-americano de Investigaciones Sociales, Academia de Guerra de la Fuerza Terrestre, Abya-Yala, Quito

Deler JP, 1981, Genèse de l'espace équatorien. Essai sur le territoire et la formation de l'Etat national, col. Recherche sur les grandes civilisations, éd. Association pour la diffusion de la pensée française, Paris

Gallegos F, 2000, La crise de l'Etat et du modèle néo-libéral de développement, in Problèmes d'Amérique latine, trimestriel $n^{\circ} 36$, janvier-mars 2000, La documentation française

Gastambide A., Sierra A., 2003, La dollarisation de l'Equateur à la loupe, Le Monde, 12 janvier 2003

Moncayo Gallegos P., 1995, Fuerzas Armadas y Sociedad, Corporacion Editora Nacional, Quito

Sierra A, 2000, “Gestion et enjeux des espaces urbains à risque d'origine naturelle : les versants et les quebradas de Quito, Equateur, thèse de doctorat, dirigée par Béatrice Giblin-Dévallet et Charles le Coeur, université de Paris VIII, septembre 2000.

Sierra A., 2005, Le risque dans la construction nationale équatorienne, in Bret B. (coord.), Intégration et fragmentation territoriale en Amérique latine, Bulletin de l'Association de Géographes Français, Décembre 2005. 


\section{NOTES}

1. En excluant l'éphémère présidence constitutionnelle de Rosalia Arteaga du 8 au 11 février 1997.

2. La définition de ce qui est à gauche diffère selon les pays mais dans la mesure où Hugo Chávez se réclame d'une forme de socialisme et qu'il a été élu en rupture au conservatisme et en dénonçant les inégalités, il peut effectivement être classé à gauche. Rappelons que le nationalisme se retrouve aussi bien à droite comme à gauche et que l'idée de nation ne peut être clivante qu'en fonction de ce qu'elle revêt (pour approfondir cf. Y. Lacoste, Vive la nation!) Mon commentaire précédent faisait plutôt référence au peu de cas que fait $\mathrm{HCh}$ des libertés démocratiques (liberté de la presse, par ex.) et pas spécialement au nationalisme, mais de fait je suis d'accord que c'est une sorte de gauche.

3. Même si les étrangers inscrits dans les consulats peuvent voter, le nombre de clandestins est tel que beaucoup d'Equatoriens n'ont pu voter.

4. La parroquia (littéralement, la paroisse) est une subdivision du canton qui ne correspond pas forcément aux municipalités surtout dans les principales villes du pays. C'est le premier niveau d'agrégation des résultats électoraux. Les résultats par bureaux de vote ne sont pas connus.

5. D'après Carlos Xavier Gutiérrez auteur d'un article dans la revue hebdomadaire Vistazo, 1er décembre 2006

6. Rafael Correa a fait ses études à l'Université Catholique de Guayaquil puis à

l'Université libre de Louvain en Belgique. Il est catholique pratiquant.

7. Ce dernier, créé au début des années quatre-vingt, a capté l'héritage de la Concertacion de Fuerzas Populares (CFP) aujourd'hui marginal.

8. Le Monde, 29 septembre 2007

9. Oswaldo Hurtado, Vice-président de Jaime Roldos, est devenu Président de la République à la suite du décès de ce dernier en 1981.

10. Président du Congrès, il avait le pouvoir de convoquer l'assemblée pour voter la destitution. Cependant, la logique institutionnelle aurait voulu que ce soit la VicePrésidente, Rosalia Arteaga, qui succède à Abdalá Bucarram.

11. Les négociations sur les concessions pétrolières et sur la lutte contre les narcotrafiquants colombiens ont largement contribué à la crise politique de 2005 et aux critiques émises par Rafael Correa à l'encontre de Luis Alfredo Palacio. (cf. site web : www.cidob.org)

12. Movimiento Patria Altiva y Soberana

\section{RÉSUMÉS}

Après 10 ans d'instabilité politique chronique, l'Equateur semble connaître une nouvelle phase à la suite de l'élection à la présidence de la République de Rafael Correa en 2006. Cet article apporte des éclairages sur la nouvelle géographie électorale du pays et sur les forces politiques avec lesquelles le nouveau président doit composer. 
After ten years of political instability, Ecuador seems to pass through a new period after the Rafeal Corea presidential election (2006). This article try to deal with the new electoral geography of this country and the different strenghts the new president have to compromise with.

Despues de diez años de inestabilidad política crónica, Ecuador parece vivir una nueva étapa tras la elección a la presidencia de la República de Rafael Correa a fines del 2006. Este artículo enfoca sobre la nueva geografía electoral del país y sobre las fuerzas con las cuales el nuevo presidente tiene que acomodarse.

\section{INDEX}

Mots-clés : géopolitique, Equateur, géographie électorale

Keywords : geopolitics, Ecuador, electoral geography

Palabras claves : geopolítica, Ecudaor, geografía electoral

\section{AUTEUR}

\section{ALEXIS SIERRA}

Alexis Sierra (alexisierra2001@yahoo.fr) est maître de conférence, agrégé de géographie, chercheur au sein de l'UR-IRD 029 « environnement urbain », spécialiste des espaces à risque et des politiques publiques environnementales en Amérique latine en supprimant tout le reste. Il est co-auteur de l'ouvrage L'Amérique latine, Velut S. (coord.), CNED/SEDES, Paris, 2005 et il vient d'éditer en collaboration avec Jérôme Tadié, Les villes face à leurs marges, Autrepart, n² 45, IRD, mars 2008. 